\title{
DISCURSO DE AGRADECIMIENTO POR LA DISTINCIÓN DE PROFESOR EMÉRITO DE LA UNMSM
}

\author{
SPEECH FOR THE DISTINCTION OF PROFESSOR EMERITUS OF SAN MARCOS \\ Óscar René Salinas Zegarra* \\ Profesor Emérito de la Facultad de Ciencias Contables \\ Universidad Nacional Mayor de San Marcos-UNMSM / Lima-Perú \\ [Recepción: Setiembre de 2014/ Conformidad: Octubre 2014]
}

Doctor Pedro Cotillo Zegarra, Rector de la Universidad Nacional Mayor de San Marcos; Dra. Antonia Castro Rodríguez, Vicerrectora Académica; Dra. Jeri Ramón Ruffner de Vega, Decana de la Facultad de Ciencias Contables; Dr. José Niño Montero, Secretario General de nuestra universidad; distinguida y apreciada concurrencia:

Dicen que fue una luz párvula de madrugada la que se instaló en la segunda cuadra de la calle Ejercicios, hoy Alvares Thomas, a dos cuadras de la plaza de armas de la volcánica Arequipa. Cuentan que así se produjo el andar del segundo de los hijos de Enrique G. Salinas Paredes y Dora Zegarra Vargas Machuca de Salinas, partiendo de un nueve de setiembre de 1940. Agradece a sus progenitores por darle la vida y a Dios por consolidársela y preservársela.

Luego, elevado a las cumbres del Misti, del Chachani y del Pichu Pichu lo fueron encontrando los pastores de la esperanza para hacerlo partícipe de los primeros conocimientos del lenguaje de los infantes en el Colegio Niño Jesús de Praga, extendido en el ombligo de la calle Mercaderes. Con esta secuela y sin pedir pausas al linaje ancestral de la siguiente entidad educativa, lo posesionan en el Colegio San Francisco. Todavía se mecen en los andariveles del recuerdo, la solemnidad de la primera comunión que recibió en una mañana asaltada por los matices de la angustia. Agradece a sus profesores y a algunos sacerdotes de ese entonces.

La calle Manuel Muñoz Najar y su tranvía coronando misticismos de recreo callejero en el distrito de Miraflores, lo hizo circular entre las tareas escolares y la casa materna para, más adelante y sin pedir permiso a las praderas, romper con estas raíces al ser trasladado al Colegio Salesianos de la Capital de la República, bordeando el pincel del año mil novecientos cincuenta. El Centro Escolar 451 de la Villa del Sol de Chosica lo estaría esperando para extenderle los confines de un machismo puritano que lo haría jinetear por andes, ríos y quebradas para concluir con obsesión el quinto año de primaria. Entonces sube al podio para agradecer a sus maestros principalmente al profesor Blanco, "Platanito" en el decir de los "coros" atrevidos.

\footnotetext{
* Doctor en Ciencias Económicas y Comerciales, Contador Público - UNMSM.

Email: oscarensal@gmail.com
} 
En una de las esquinas del parque central chosicano yacía el Colegio Nacional Pablo Patrón, enfundado en gruesas rejas, puertas y ventanas el mismo que, prestado o alquilado, servía de día para un estudiantado de primaria para que, con los estertores de la noche, irse convirtiendo en la secundaria nocturna destinada a una juventud desprovista de paternalismo. Gracias profesores en las figuras de "Pelito"( Matemáticas) y "Significado Almeyda" (Lenguaje y Literatura) en esos dos primeros años de la media común.

El río hablador deletreaba sus pausadas aguas por la hendidura de una rivera posesionada en los linderos de la Escuela Normal Superior Enrique Guzmán y Valle - La Cantuta en lenguaje no cristiano - y su Colegio de Aplicación para estudiantes secundarios. Allí recibe los tres últimos años de este ciclo asido a profesores de la talla de Manuel Moreno Jimeno (Castellano y Composición Literaria), Carmela Rocha (Química y Física) y Ernesto Viacava Rocca (Algebra, Geometría y Trigonometría) con quien por designios inexplicables se viene juntando ahora en la Academia Nacional de Ciencias Económicas. Gracias en todos los sentidos.

Asomar su cuerpo y su alma al intento de ingresar a San Marcos sembró de horas compulsivas las fases del mismo. Luego de aprobar el escrito se enfrentó en el oral a tres descendientes de los justicieros de la Santa Inquisición. En una tarde otoñal, bordeando a hurtadillas la cara del destino, se acercó a la espectral vitrina amasada por un tumulto casi agónico. Colgar la mirada en la letra "S", suspirar asfixiadamente y ponerse en brazos del éxtasis al percibir sus apellidos y nombres como "ingresante", hizo eclosión en el dique emocional contenido con esfuerzo. No tuvo aliento ni fuerzas para repeler el "bautizo a los cachimbos" que recayó sobre sus cabellos, los que fueron rodando en mechones atragantados por los acordes de un sinnúmero de tijeras.

Protagonista consuetudinario de los estudios emprendidos, los días, meses y años lo llevaron a posesionarse de la especialidad contable como integrante de la Promoción 1963 "Alberto Merino Llanos" con quienes el pasado mes de Noviembre ingresó a la estela de los cincuenta años -bodas de oro-, hasta lograr el título de Contador Público en el 65, sin descuidar los estudios de doctorado en ciencias económicas y comerciales que lo llevaron a ese grado en 1973. Gracias a los profesores de esa época dorada en las efigies de de Victorio Vallo, Juvenal Santa Cruz y Mario Ordóñez. Un agradecimiento especial a los integrantes de su Promoción que lo acompañan. Gracias Luis Vargas, presidente por siempre.

Encontrarse preparando una clase, ingresando a un aula, recibiendo el saludo de los alumnos y sintiendo el raspado de la tiza en el lomo de la pizarra, fue como un síncope que estaba por acontecer. La Academia de preparación para el ingreso a la universidad, patrocinada por el Club César Vallejo de Chosica del cual era ya su Presidente, lo introdujo en esta temática. Loas para los que caminaron junto a él, enfrentando una adolescencia de contrastes generacionales.

Chiclayo, la polvorienta Chiclayo, lo recibe en los anaqueles de la entones Universidad Nacional de Lambayeque, hoy Pedro Ruíz Gallo, para examinarlo a través de un jurado de excepción entre los que se contaban a Luis Bramont Arias y Roberto Keil Rojas y hacerlo ingresar por la puerta grande a la docencia universitaria en el año 66. Lejos estaba su imaginación de que esta casa de estudios, años más tarde, lo haría coger el emblema de Profesor Emérito. Luego de cimentar esta antorcha, regresa a Lima y se inserta en la Universidad Peruana de Ciencias y Tecnología - La Tecnológica en el lenguaje cotidiano- ahora Ricardo Palma, para cumplir cinco años de exigencias plenas. Extiende un agradecimiento especial a esas dos universidades.

La dicotomía del año 67 lo encuadran dentro de la Dirección General de Contribuciones, hoy SUNAT, como Auditor Impositivo. Si querer es poder, entonces tuvo que pasar por la exigencia de dos nombres que con el tiempo enarbolarían la bandera de la profesión contable: Juvenal Santa Cruz y Simeón Caballero. Pero, ¿en cuál de las esquinas de la historia lo acechó San Marcos para poseerlo como su servidor imperecedero? Entre lágrimas y brisas del inicio de la década de los setenta, lo ven ingresando por la primera cuadra del jirón Ayacucho, calle Tigre a decir de los historiadores taciturnos, y trepidar una casona para ser entrevistado por el Jefe del Departamento de Contabilidad y Administración de la universidad más antigua de América. La misma casona, de tránsito provisional mientras se terminaba el pabellón en la Ciudad Universitaria, lo recibe en su clase 
inaugural. Posesionarse del pupitre, la pizarra, la tiza y las miradas inquisidoras de los alumnos, cinceló todas las alegorías anudadas en su respiración artificial. Paralelamente, la Cía Goodyear del Perú lo integra para que preste sus servicios profesionales, lo que no es óbice para que sustente su tesis de doctorado intitulada "Comportamiento de los Costos Aplicados en la industria Peruana del Cuero". Un agradecimiento sin límites a los docentes que lo examinaron y apoyaron en la persona del Dr. Celestino Jara, Jefe del Departamento mencionado.

Y es en este Departamento primigenio de la legendaria San Marcos que se produce el encuentro con la poseedora de unos ojos almendrados de púrpura azucena y de una cabellera cuya vertiente provenía de algún maizal espectacular: Elsa Gordon. El "sí, padre, acepto" los unió para toda la vida un cuatro de setiembre de ese inefable 76. Gracias inconmensurables para la compañera eterna de su vida que trajo entre su preciado equipaje a una mujer que se constituyó en el talismán de todo lo vivido: La monumental "mamá Charo", "la can" a decir de sus hijos, a quien extiende su enorme gratitud. Y es así que, en el 77, Oscar Alberto baja la bandera como primogénito para que se instaure la dinastía Salinas Gordon. Este acontecimiento sirve de preludio para que en los albores de ese mismo año conforme el grupo ganador de la directiva del Colegio de Contadores Públicos de Lima.

Como un fogonazo de posición meridiana, el inicio de la década de los ochenta le trae a su regazo al segundo de la dinastía de nombre José Manuel para que conjugue con los verbos avanzar y lograr. La ahora Facultad de Ciencias Contables le concede el ascenso a Profesor Asociado y la Empresa Comercializadora de Alimentos S.A. lo posiciona en cargos versátiles de inconmensurable responsabilidad. Once años se inoculan en el torrente sanguíneo de las tareas asumidas. Gracias a todos los que colaboraron con él.

La misma década hace caer otro trozo del almanaque al ampliar la dinastía en Noviembre del 81 recogiendo los gemidos de Elsa Patricia y trasladarlos a la Dirección de Actividades Profesionales del Colegio de Contadores Públicos de Lima donde lo señalan como integrante de una nueva directiva. Parado junto al trueno de esos años, recibe en su ser el complemento de la dinastía adscrita por el todopoderoso: la llegada de Elia Paola en febrero del 83. El ascenso a Profesor Principal en el 86 es el preludio para llegar, en el 87, otra vez a la directiva del Colegio a desempeñar, esta vez, el cargo de Director Secretario. Gracias mil a los caminantes que lo acompañaron por esa senda. Gracias a sus hijos por hacerlo inaugurar, a cada instante, el deseo de vivir.

Los noventa y más, lo sumergen en la directiva de la Federación de Colegios de Contadores Públicos del Perú llegando a Buenos Aires a participar como Panelista en uno de los grupos de trabajo de la Conferencia Interamericana de Contabilidad. Mientras tanto, en la Facultad se producía la elección de un nuevo Decano, bajo un cielo algo encapotado. La propuesta, el conteo de votos y su elección constituyeron para él un epicentro de envergadura insospechada. Presidir los Consejos de Facultad así como asistir a los Consejos Universitarios y a las Asambleas, extendieron sus responsabilidades al interactuar con las autoridades de turno como el Rector Wilson Reátegui y los Vicerrectores Piscoya y Anaya. La proa hacia el éxito se encopetó al interactuar con la historia y los nombres de otros decanos que lo acompañaron: Pedro cotillo ( Farmacia), hoy mirándolo desde su asiento de Rector, Juan Espinoza (Veterinaria), actualmente ejerciendo la jefatura del Centro Preuniversitario, Jaime luyo (Electrónica) y Carlos Ponce (Sicología), amigos por siempre, entre otros. Gracias enormes por todo lo vivido, gracias señor rector por haberle facilitado llegar a estos momentos.

Abriendo de par en par las cimbreantes y achocolatadas aguas del Amazonas a bordo del legendario "Juliana", lo encuentra el año 92, compartiendo con profesores y autoridades de las Facultades y de la AIC, un congreso de docentes aclimatado en Iquitos. Para variar, la Facultad Sanmarquina y su Decano son electos para dirigir la Asociación Nacional de Facultades de Ciencias Contables y Financieras -ANFACOFI- por un periodo de dos años. Producto de estos acontecimientos, le encargan la elaboración y exposición del trabajo interamericano a ser presentado y expuesto en la siguiente Conferencia interamericana de Contabilidad a escenificarse en la paradisiaca República Dominicana, en un irreversible 1993. 
Unificar la currícula a nivel nacional fue tarea de titanes así como la modernización Académica y Administrativa de la Facultad incluyendo la biblioteca, Hemeroteca y el primer laboratorio de cómputo. El vuelo del tiempo lo inserta en la continuidad de tareas llegando a ser propuesto para la reelección en un no tan apacible mes de Octubre del 94. Recibir un segundo mandato lo impregna de mayores responsabilidades, al continuar también en la presidencia de ANFACOFI y recibir el encargo de la organización del II Congreso Interamericano de Profesores del Área Contable a escenificarse en Lima en setiembre del 97.

Las gotas de cristal que se desprendían de un cielo sonrojado de entusiasmo, se oscurecieron con el repiqueteo de las aves negras. Un aciago mes de mayo del 95, la sinrazón de la política gubernamental, decretó la intervención de San Marcos con la secuela de que todas sus autoridades tenían que poner sus cargos a disposición. Marcar con paso firme las vicisitudes de esos días compulsivos, lo llevaron a tomar la decisión de pedir su cese voluntario al tener la acumulación de los años de servicio requeridos. Esta coyuntura lo lleva a patentizar el nacimiento de Rosas Salinas Hernández y Asociados, Contadores Públicos Auditores Asesores de Negocios, firma que se viene empinando hasta nuestros días y a integrarse como Perito Contable Judicial de la Corte Superior de justicia de Lima. Agradece a sus socios Atilio Rosas y Aníbal Hernández por compartir las vicisitudes cotidianas. Gracias al Comité de Peritos del Colegio de Contadores Públicos de Lima.

La incongruencia no pudo detenerlo. Escenifica el Congreso interamericano de docentes del área contable ya programado colaborando, además con las Universidades Garcilaso de la vega y Nacional del callao en programas para la obtención del título profesional de Contador Público. Pero al no haber mal que dure cien años ni cuerpo que lo resista, la bajada de bandera del 2000 cincela el renacer de San Marcos retornando a su hábitat con mayor gloria e irreverencia ante la adversidad. Este retorno lo sitúa en los cursos de Posgrado de la Facultad con una continuidad que todavía se mantiene. Gracias a los directores de esa pujante Unidad, gracias doctor Alejandro Flores, director de turno. Sin dar tregua al cansancio, se abre otra puerta para introducirlo en el doctorado de Contabilidad y Administración de la Universidad Nacional Federico Villarreal llegando a enarbolar ideas y conocimientos por más de una década. Reconoce también a los directivos de esa casa de estudios.

La Universidad Continental de Ciencias e Ingeniería emboscada en la incontrastable ciudad de Huancayo lo posesiona como profesor y decano de la Facultad de Ciencias de la Empresa sintiendo la brisa del 2003 hasta un atardecer del 2006. Confabulado con este tipo de tareas recibe en Lima la sintonía de la Universidad Peruana de Ciencias e Informática para fustigar el cargo de Responsable de la Carrera de Contabilidad y Finanzas partiendo en dos el periplo entre el 2007 y 2008. Empinándose sobre los cimientos de estas dos casas de estudios, las involucra también en su agradecimiento pleno y total.

Recibiendo el sol y bajo los paraguas abiertos a la trascendencia profesional y personal, recorre también las letras de molde de dos Revistas: El Contador Público y Actualidad Empresarial con artículos sobre costos y gestión empresarial interviniendo como expositor en algunos seminarios que lo perpetúan en los caminos transitados por los asistentes de turno. Su itinerancia lo lleva a participar como ponente en "Tributa 2009" en "Abancaycito" con el tema "Incidencia de los Tributos en los Costos Empresariales". Un agradecimiento especial a Alejandro Rojas y a Melisa Fernández, iconos y directivos de Actualidad Empresarial y a la Federación de Colegios de Contadores Públicos y su Junta de Decanos.

Dicen que las generaciones intelectuales se suceden unas a otras en la gran contienda que viene librando el hombre por arrancarle al universo sus secretos y sus misterios. La suma de estas generaciones se agolpan en su mirada evocando cada clase, cada diálogo, cada instante en que cabalgaron juntos por las vertientes del conocimiento. El reloj de la vida lo catapulta a acofradarse en ellas para agradecerles en grado superlativo por haberle permitido hacer un camino conjunto.

La década del 2010 lo viste de una armonía afinada en el espacio. Significa traspasar toda una suerte de propósitos como el ser propuesto como profesor emérito por el Consejo de Facultad y sus Decanos de entonces. Un agradecimiento especial a los doctores Alfonso Ugarte y Alejandro Flores forjadores de este galardón

212/ QVIPURAMAYOC | Vol. 22(41) 2014 
conjuntamente con quien lo continuó y materializó: La doctora Jeri Ramón, actual decana, para quien entrega su reconocimiento más efusivo. Presidir la comisión organizadora de los 50 años de su promoción en el pasado mes de noviembre lo pone en una ruta insospechada ya que sería en este mes de vivencias excepcionales que se ilumina el firmamento con el amanecer de Emilito Zegarra Salinas, su primer nieto, para decirnos con su "Agú" que su presencia trasciende los albores de cualquier dinastía adosada en su pequeño mundo. Pero el mundo es ancho y ajeno a decir de Ciro Alegría; es el ir y venir por estos corredores de pasos centenarios y de intelectualidad sin límites. Es mecerse en los cauces de la historia en este salón general, donde lo ven de pié, los mira y hunde sus sentimientos en cada uno de los presentes descalzando las ideas, lanzándoles un agradecimiento integral que traspase cualquier alegoría arropada en un emblema. Entonces pide, con humildad, que le permitan balbucear las célebres frases de aquel poeta catalán y decirles que "En estos momentos él quisiera ser tan grande, tan grande y poder ver el mundo a través de los ojos de un niño”.

Muchas gracias. 